\title{
Towards An Adaptive Multimedia Learning Environment
}

\author{
Frank Kurzel \\ University of South Australia, Magill, \\ Australia
}

\author{
Jill Slay and Yenha Chau \\ University of South Australia, \\ Mawson Lakes, Australia
}

\section{Frank.Kurzel@unisa.edu.au Jil.stay@unisa.edu.au Yenha.Chau@unisa.edu.au}

\section{Abstract}

In this paper, we describe an evolving adaptive multimedia learning environment that utilises multimedia presentation techniques in its interface while still providing Internet connectivity for management and delivery purposes. The structure of the domain is based on concepts that can be presented in a variety of ways; multimedia players display the content on the client computer. A student and course model exists that determine what content item is presented, based on both student prior competencies (pre-requisite knowledge and skills) and preferences.

Keywords: Educational multimedia, Adaptive Systems, Dynamic course generation, Student profiles.

\section{Introduction}

With the problems imposed by large classes, and the large range of individual approaches needed to deal with some of student learning issues raised above, Multimedia academics have been some of the first to develop and use web-mediated learning environments for enhancing student learning. As has been pointed (Slay 1998a; Slay 1998b) the World Wide Web (WWW) provides a vehicle for the development of the learning environment and teaching can be structured to develop lifelong learning skills and to cater for the expectations and learning styles of students with a wide range of backgrounds.

Early Australian examples of the use of the WWW in IT education abound. Boalch (Boalch 1996) provides an examination of the use of the WWW as a support medium for the delivery of a first year unit in Information Systems at Curtin University. He provides an evaluation of site utilisation and user feedback in the case where subject information and course details were provided on the WWW for students. The Eklunds (Eklund and Eklund 1996) examine the use of the WWW to supplement traditional IT teaching. They provide case studies of two examples of the re-structuring of traditional forms of IT course for Webdelivery. Jones (Jones 1996) of Central Queensland University gives details of case study involving the design, presentation and evaluation of an undergraduate unit in Systems Administration taught completely via the WWW to on-campus and distance students.

However these and many newer systems that have been developed rely on the low-level concept of interactivity (derived from a distance education paradigm) as the relationship between an individual student and text, and fail to use the technological interactivity, which is available. Common online learning envi-

Material published as part of these proceedings, either on-line or in print, is copyrighted by Informing Science. Permission to make digital or paper copy of part or all of these works for personal or classroom use is granted without fee provided that the copies are not made or distributed for profit or commercial advantage AND that copies 1) bear this notice in full and 2) give the full citation on the first page. It is permissible to abstract these works so long as credit is given. To copy in all other cases or to republish or to post on a server or to redistribute to lists requires specific permission from the publisher at publister@intormingscience.org ronments often fail to maximise the potential of current multimedia resources. While the value of HTML pages and threaded discussions is acknowledged, they do not display the ability to adapt teaching material for the needs of individual students. 


\section{Adaptive Teaching on the WWW}

Various researchers who have experimented with adaptive teaching on the WWW have used techniques and principles derived from Intelligent Tutoring Systems (ITS) and particularly Anderson's rule based cognitive modelling (Anderson 1983). Others have used Adaptive Navigation Support (Brusilovsky 1996) to provide adaptive navigation through hypertext pages and thus developed adaptive textbooks for the tutoring, particularly, of software applications (Brusilovsky 1996).

An ITS is traditionally formed of two parts, an expert system and a communication module. Within the expert system, there are three modules, the student module, the pedagogical module and the domain knowledge module. The student module gives the student history; the pedagogical model is provides information about the teaching process and the domain knowledge module provides the material that is being taught. ITS have been shown to enhance student motivation and learning (Anderson 1983). While proving useful, adaptive teaching on the WWW has not yet been able to supply the full functionality of traditional ITS.

This paper presents techniques that have been used to provide adaptivity within Multimedia Concepts http://mm.unisa.edu.au, an introductory course within the multimedia studies stream offered at the University of South Australia (http://wWw.unisa.edu.au. The aim of this course is to provide the foundational knowledge and skills required to create and utilise a range of media items within multimedia presentations. The students come with a diverse range of knowledge and skills. The course is situated with an Arts degree, but a large number of students from Computing, Education, Art/Design to name a few, undertake it. These students can also come from different levels/years in their study programme.

With the movement of courses to an on-line mode, there was a need to develop and enhance the learning environment with a range of features that stimulate the complete learning experience. We have developed a prototype for a multimedia learning environment for the presentation of this course that displays adaptive characteristics and has become an Adaptive Multimedia Learning Environment. (AMLE). The aim is to develop the full functionality of a traditional ITS that supports other domains.

\section{Implementation of the AMLE}

The advantages of this delivery format of learning materials include firstly, the greater flexibility for the student in pursuing the course; the materials are available at the students' convenience and staff have convenient on-line access to all course management facilities within the course. Accommodating new students with a range of backgrounds is often a problem for both the learner and the teacher in standard teaching situations. An adaptive system that makes available specific content (adaptive presentation) (Brusilovsky 1996) provides students with a format of the new knowledge and skills appropriate to the students' level of understanding. Alternately, the system might provide links to appropriate content (link level adaption) (Brusilovsky 1996).

The technology supporting hypertext and hypermedia systems have been with us for some time. The use of a range of interactive multimedia materials to support the learning is seen as a major motivation; these media rich systems have the potential to enhance the student learning in very positive ways. In providing the students with a range of media forms and tasks to satisfy, we are accommodating different learning styles. An adaptive system has the potential to monitor student usage of different modules and respond accordingly. This episodic adaptivity will provide real time modification to any survey generated preferences gathered at the beginning of the course.

We have developed this multimedia learning system using Macromedia Director, ColdFusion, HTML and CGI Perl script that allows rapid prototyping and use of various teaching strategies as alternate paths through the presented material. 


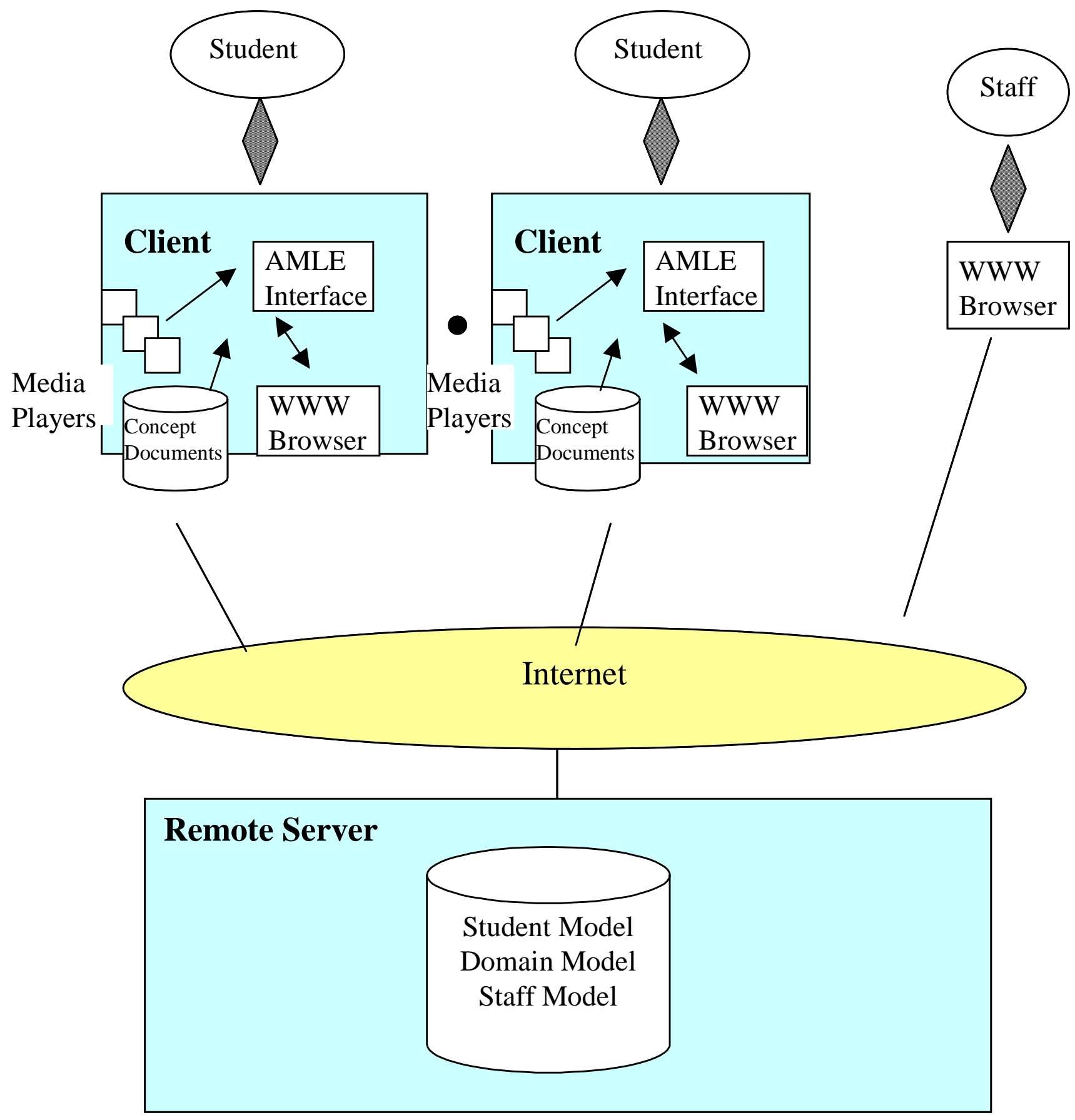

Figure 1 AMLE Architecture

\section{AMLE's Architechture}

AMLE provides multiple access to course materials for students along with course management materials for staff over the Internet. The student (or client) works with course materials through an application that interacts with a remote server. It also accessies locally stored documents files. A number of players are provided on the client side which are activated as a result of student and domain model data distributed by the server. Staff/tutors interact with the course and student model through a WWW browser. Media files are stored locally but administered over the WWW. 


\section{Interface Details}

The main interface provided for students to access the learning materials can be classified as a hybrid browser. It takes advantage of the multimedia development environment and enables a range of concept players to be used depending on content availability and student preference. These range from a concept being displayed as text formatted with HTML tags, to a concept being displayed as an acrobat postscript file, containing both textual and graphical information.

Video and audio formats can be utilised, along with appropriate animations. A programming player exists to display the result of an execution, with the appropriate programming feature described and displayed. A range of other appropriate tools are provided in the interface to enhance the learning environment. For example, on-line tutorial groups can be established with the ability to discuss particular content-based issues. Tutors can address any problems that are not accounted for by peer group interactions through the provision of and electronic helpdesk.

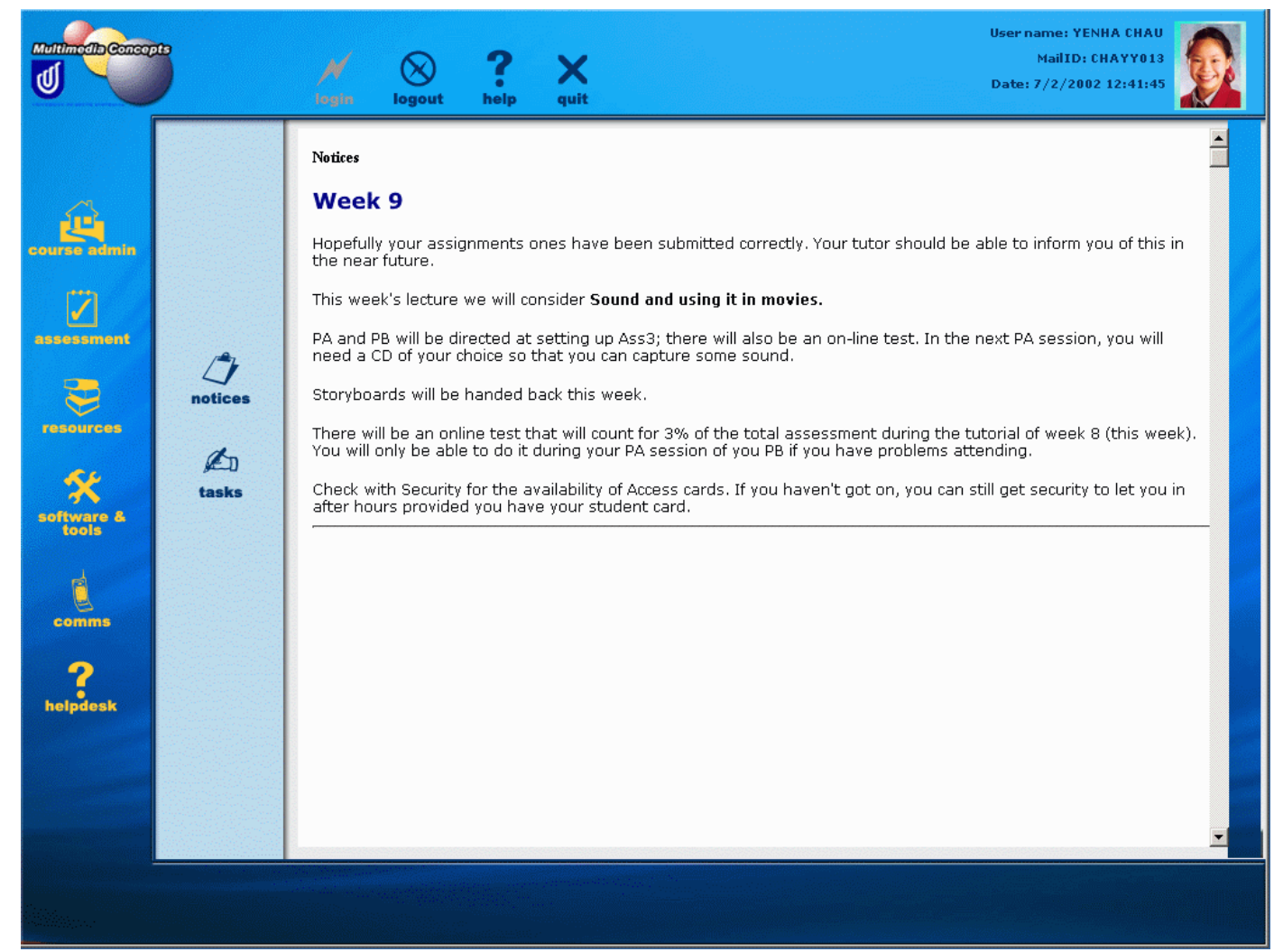

Figure 2 Main Interface

The main interface is a windows environment that initially provides authenticated logging on/off facilities. Once identified and registered, the user is provided with the current session, or the pre-test for that session if this is the first time in. From there, individual concepts can be accessed through a session player. Sessions are groupings of concepts and tasks that the domain expert has predefined for the course. This would correspond to the content provided in a weekly lecture(s) and associated tutorial/practical activities 


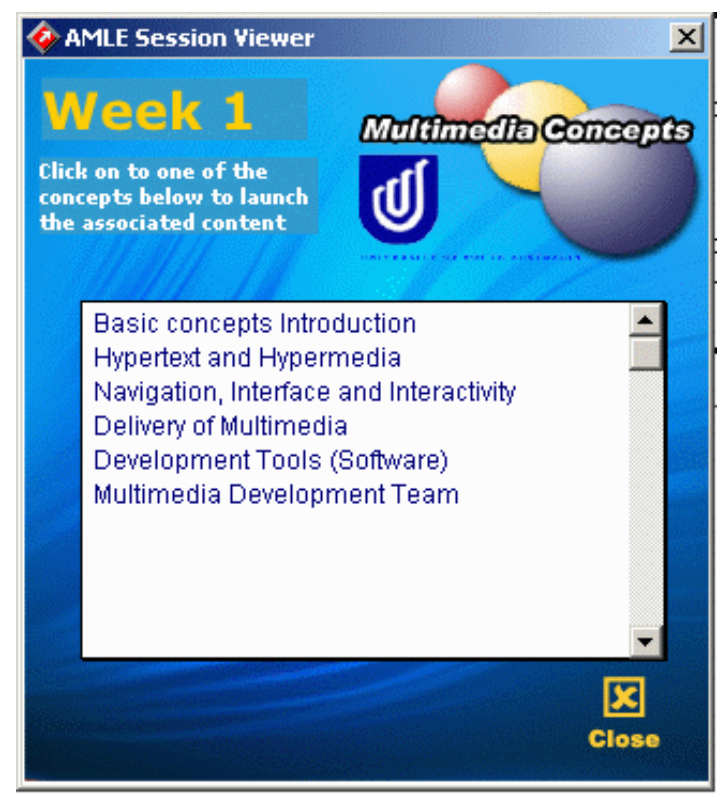

Figure 3 Session Viewer

It has been argued that link anotation (Brusilovsky, Eklund et al. 1998) provides the user with extra information about the content available, enabling a more informed decision. We employ annotations within the session viewer to indicate the content that a mouse click will provide. Where the system can infer that the student knows the concept or can perform the task, the session viewer is annotated accordingly. We chose not to hide this link so that the student is still able to follow it for revisional purposes perhaps.

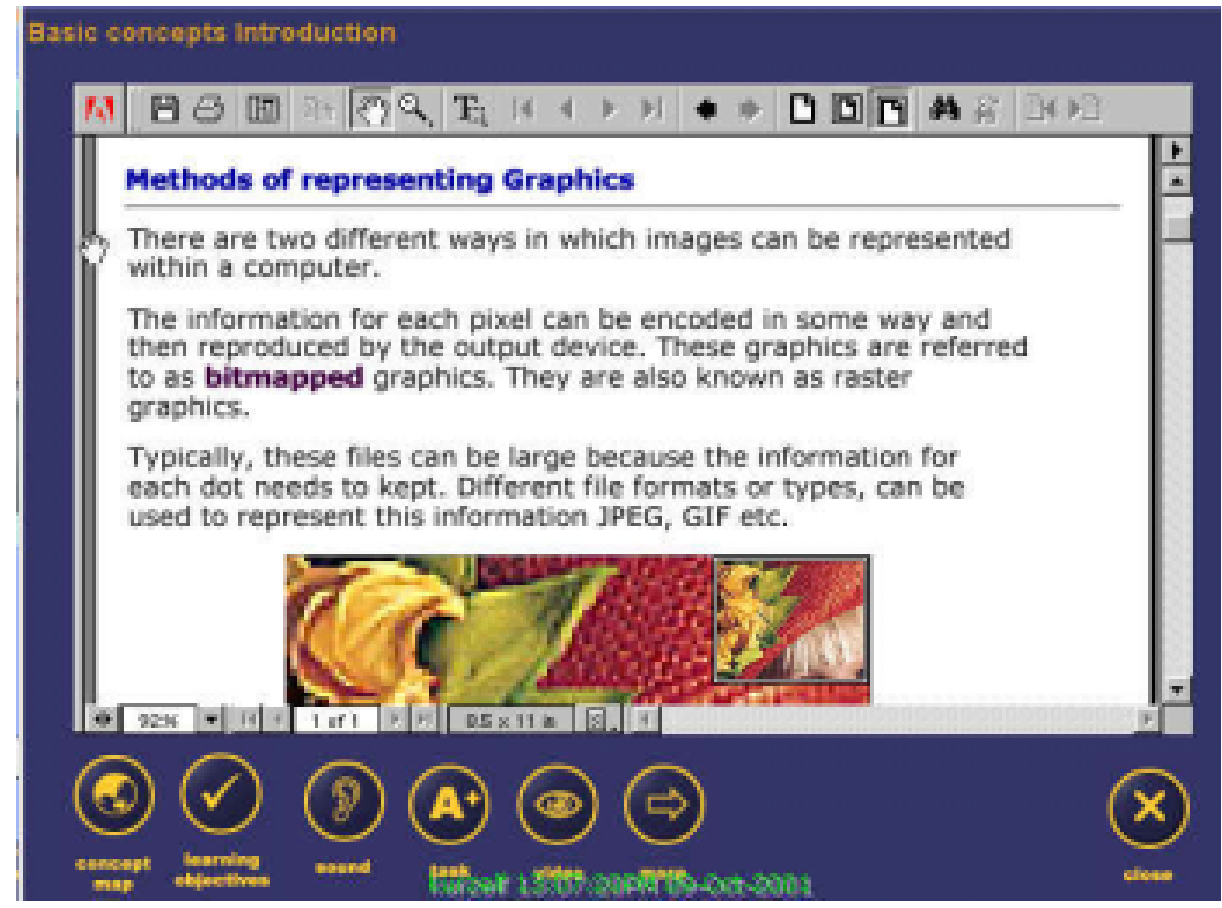

Figure 4 The default player

The default player is one that displays the content in pdf format. We chose this to ensure that the content (images and text) could be guaranteed while still providing WWW facilities for hypertext links in the documents. 


\section{Domain Knowledge}

Our course model is organised hierarchically into courses, sessions, concepts and documents. Sessions have been defined from concepts; sessions can have any number of concepts but a concept can only appear once within any session of a course. A concept consists of many documents; a document can be the content of a concept, its objective, any pre-requisites, and assessment criteria; alternately, it might be a problem to be solved. There is a player associated with each document that displays the information in an appropriate manner. For example, the learning objectives of the concept are presented in a textual form within an additional window. An alternate presentation format might be audio. Players supporting this functionality are located with the client.

We have treated our documents as child-pages similar to hanging-pages in (Murray, Condit et al. 1998). Each document can exist in multiple media formats that include text, video, audio, HTML files, Director movies and Acrobat pdf files. The list of documents needed for a concept is stored in AMLE Microsoft Access database and is stored on a remote server. We have an 'Authoring Tool' for lecturers to maintain the course model; course content is stored on the client side to ensure quick delivery. We argue that content does not change over the duration of the course and does not need to be delivered dynamically over the WWW.

Our implementation is supportive of other possible domains of knowledge; the delivery system then is content independent. Tools for both the generation and subsequent use of the system have been investigated and an online database supporting both the student and course models, has been established. The placement of content into well-defined modules, provides a mechanism for the flexible reuse. The course metadata is stored on a remote server along with the student profile information. We utilise an overlay model (Brusilovsky) and allow WWW access to this information; sections specifying personal preferences can be viewed and amended by the user.

As mentioned previously, the content is organised as a number of concepts and/or skills that need to be satisfied. To accommodate a range of students at various levels in their programmes, we pre-test at the session level. Another alternative considered and still to be investigated, might be to pre-test at the course level and infer knowledge from the results. For the practical components, students might be able to demonstrate the possession of some particular skills; this then needs to be handled manually and/or electronically.

The content of the delivery system is relatively static; that is, the teaching components that are delivered through a range of players accommodating text, pdf modules, video, audio, animations, etc. and any other media format that might become available in the future, are stored at the client side. The dynamic components of the delivery system involving the course structure and assessment pieces, student profiles and assessment results, episodic data mapping student usage, and data of those on-line, is stored on the server side.

This 'dynamic' data allows the system to indicate to the users, information about the concepts/tasks that they have covered. It also provides them with a current 'report' of results of assessment pieces. The registration of students and tutors online, coupled with profiling information, enables the establishment of online groups that reflect the stored data; these groups could be for example, assigned at the expertise level or by any other criterion considered educationally worthwhile.

A number of 'hanging pages' in the concept player provide information about the learning outcomes of the concept and alternate presentation mechanisms eg video, audio. Assessment tasks are specified at this level, as are pages of further explanation. The system utilises the student profile information, greying out options that are not required. Other tools that are used that might be used in the learning process eg email, chat facilities, submission mechanisms, glossary, search engine etc. along with more general facilities like audio players, WWW television, CD player, notepad etc are provided. 


\section{Student Administration}

Similarly an on-line instructional management system has been constructed to establish courses based on concepts and sessions. We have an 'Administration Tool', which enables lecturers and tutors access to our student model. Lectures can add/delete a tutorial group, add a new student, search for existing student, andupdate student's detail such as tutorial scores, assignment scores and exam scores. Tutors have access to update and search facilities of students' tutorial and assignment details.

AMLE uses and maintains student profiles that contain a summary of the student's past experiences and other preference information. The course model and student profile allows students to proceed at their own rate through the learning environment with this access being influenced by a student's competencies and preferences (i.e. the student history of the traditional ITS).

The management system enables tutors to enter marks electronically and subsequently manage the assessment components of the course. Reporting mechanisms then cope with student, tutor and course co-ordinator requirements.

\section{Current and Future Work}

Our major concern is to provide adaptivity and interactivity for our students since we recognise that our students' backgrounds and cultures have given us a wide range of learning styles and expectations among our students. Currently, we have created a range of hybrid browsers with varying interfaces to access the course model; a number of players have been created to display the content. These players are dynamically activated, depending on the page type. The student's history and preferences will be fully developed in future implementations. A range of tools including tutorial chat facilities, notebooks and email, are also provided in the interface for the users to take advantage of. Further work will involve the pre-testing of students and the full utilisation of student preference data collected.

Currently, the materials or concepts have been grouped into sessions that correspond to a multimedia topic that could be covered in a week. The content for each concept can be accessed sequentially; a range of tests and tasks has been generated to ensure that the student understands the concept, or is able to complete an associated task. The monitoring of these competencies is conducted by the delivery system with the student competency profile being updated as the student accesses each task. For tasks that require demonstration, the tutor can record these competencies within the student competency profile via a WWW interface. To allow for prior knowledge, students are pre-tested at the session level.

A range a questions and question types are randomly presented and marked by the system; these results then form the basis of the student's competency model. Tutors are able to enter marks directly into the student competency profile for tasks where the competency for a concept can be demonstrated. The student's competency profile is further enhanced by an assessment profile that includes summative activities that could extend over a number of sessions. Both profiles are available to the students through a WWW browser interface that displays their current competency and assessment states.

The concept structure of the course materials does not then necessitate that students access materials in this sequential manner. A number of instructional methodolgies could be employed on a weekly (session) basis, or alternately at the assignment level. Weekly tasks might be assigned that require the student to create some multimedia object for example.

We might be introducing the topic of Sound in Multimedia with the practical task being to create an MP3 player. Areas to be addressed would include:

- the possible application of sound in multimedia applications;

- theory of sound - representation, capturing, etc; 
Towards An Adaptive Multimedia Learning Environment

- sound formats - compression, filetypes, size etc.

- $\quad$ sound capture;

- $\quad$ sound control within multimedia applications;

- ethical considerations re: copyright etc.

The extension of this is to use the materials based on larger assignments that also address similar areas and could include summative tasks incorporating prior knowledge eg. incorporating images and textual information within the player.

We are establishing student/student and tutor/student interactions via online discussion groups. Students can opt to be assigned to a tutorial discussion group with a number of their peers currently logged on. The number participating in these discussion groups is 4 by default; however, this is a system parameter and can be changed. Students are initially allocated on a first in, first allocated basis but any part of the student profile could be used for this purpose. For example, students could have their own preference list, or might be assigned using academic or cultural information.

There also exists an online helpdesk that simulates a tutorial helpdesk situation where students pose questions to tutors who are currently on line. A range on tools will be implemented to facilitate these discussions including an electronic blackboard, which will allow tutors to broadcast solutions, graphics, scripts etc to help with any explanation.

\section{Conclusion}

We have established and multimedia learning environment that adapts to prior knowledge through the use of link annotation; students can also specify the type of media presentation they prefer to use. The dynamic components of the learning environment i.e. assessment, course structure, instructional methodologies etc. are stored on a remote server and are accessed through the WWW. Tutors have convenient on-line access to student groupings to enable marks entry.

We utilise two forms of assessment; namely formative assessment that includes the mastery (or competency) of particular concepts or skills conducted generally at a weekly level, and summative assessment that necessitates the use a range of knowledge/skills in its satisfactory completion. These are recorded within the ovelay model of the student profile.

A range of other tools like search engines, indexes, helpdesk, tutorial groupings etc. allow the lecturer to employ a range of teaching methodologies to satisfy the requirement of the course.

\section{References}

Anderson, J. (1983). The architecture of cognition. Cambridge, MA.

Boalch, G. (1996). WWW as an Educational Support Medium: An Australian Case Study. AusWeb96, Gold Coast, Australia.

Brusilovsky, P. Efficient Techniques for Adaptive Hypermedia. Intelligent Hypertext:: Advanced techniques for the World Wide Web, Lecture Notes in Computer Science. C. Nicholas and J. Mayfield. Berlin, Springer-Verlag: 12-30.

Brusilovsky, P. (1996). Integrating Hypermedia and Intelligent Tutoring Systems: From Systems to Authoring Tools. Proceedings of AIED.

Brusilovsky, P. (1996). "Methods and Techniques of Adaptive Hypermedia." User Modeling and User-Adapted Interaction Vol 6(2-3): 87-129.

Brusilovsky, P., J. Eklund, et al. (1998). "Web-based Education for all: a tool for development adaptive courseware." Computer Networks and ISDN Systems 30: 291-300.

Eklund, J. and P. Eklund (1996). Integrating the Web and the teaching of technology: Cases across two universities. AusWeb96, Gold Coast, Australia.

Jones, D. (1996). Solving some problems of University Education: A Case Study. AusWeb96, Gold Coast, Australia. 
Murray, T., C. Condit, et al. (1998). A Preliminary Framework for Concept-Based Adaptive Hypermedia. ITS-98 workshop on WWW-Based Tutoring.

Slay, J. (1998a). Using the World Wide Web to Create Foundations for Lifelong Learning - An Australian Perspective. Teleteaching, IFIP World Computer Congress98, Vienna.

Slay, J. (1998b). Using the WWW to Create an Effective Cross-Cultural Learning Environment. Hong Kong Web Symposium 98, Hong Kong University.

\section{Biographies}

Frank Kurzel is a lecturer in the School of Communication, Information and New Media of the University of South Australia. He has been the Program Director for the Multimedia Studies Major within the Bachelor of Arts program at the University of South Australia. He has had extensive experience in Education, Computer Science and Multimedia areas.

His research interests include web-based instructional systems to support his teaching, and the integration of Intelligent Tutoring Systems technology into hypermedia environments. He is also interested in instructional methodologies and enhancing the educational environment.

Jill Slay is a Senior Lecturer in the School of Computer and Information Science of the University of South Australia. She is currently Director of International Programs within the school and manages the Offshore programs taught in Hong Kong and Malaysia.

She has an interest in IT security education, and is publishing in this area as well as in information warfare and more generally within complex sociotechnical information systems, particularly C2 systems. She is also carrying out research in e-commerce and adaptive web-based multimedia systems and supervising research in the technical and pedagogical design of online and multimedia learning environments.

She leads the cross-disciplinary Technology in Higher Education Research Group and also works within the Advanced Computing Research Centre and the Systems Engineering and Evaluation Centre.

Currently she is Deputy Chair of the University Women's Consultative Committee and co-chair of the Division of IEE Equity Committee and as such is a member of the Division of IEE executive.

Yenha Chau is a master student in the School of Computer and Information Science at the University of South Australia, Australia. Her interests in adaptive hypermedia, Intelligent Tutoring System, student modelling and multimedia adaptive user interfaces led her to carry out her research in adaptive multimedia learning environment. In this context she contributed to developments in AMLE's student model, domain model, staff model, AMLE's tools and the adaptive multimedia user interfaces. 